Revue européenne des sciences sociales

European Journal of Social Sciences

$\mathrm{XL}-123 \mid 2002$

La société de l'information. État des lieux

\title{
La technique comme fiction
}

\section{Lucien Sfez}

\section{OpenEdition}

\section{Journals}

Édition électronique

URL : http://journals.openedition.org/ress/612

DOI : $10.4000 /$ ress.612

ISSN : 1663-4446

\section{Éditeur}

Librairie Droz

Édition imprimée

Date de publication : 1 juin 2002

Pagination : 65-74

ISBN : 2-600-00693-1

ISSN : 0048-8046

Référence électronique

Lucien Sfez, "La technique comme fiction », Revue européenne des sciences sociales [En ligne] XL-123 | 2002, mis en ligne le 03 décembre 2009, consulté le 01 mai 2019. URL : http:// journals.openedition.org/ress/612; DOI : 10.4000/ress.612 


\section{Lucien SFEZ}

\section{LA TECHNIQUE COMME FICTION*}

Technopolis est un faisceau de discours de fiction. C'est la fiction, croyonsnous, qui est le moteur de la technique, et par là de la politique. La fiction (celle du progrès par exemple) exerce une séduction dont nous sentons nous-mêmes les effets dans notre vie quotidienne, sans savoir que ce qui nous paraît le plus sérieux appartient souvent au régime de la fiction.

Attention: fiction ne veut pas dire illusion. La fiction n'est pas hors de la réalité. Elle se réalise à tous moments. Nous l'appelons quelque fois utopie, bien obligés de reconnaître après un moment que cette utopie s'est réalisée et devient un élément de la réalité. Ou encore, on peut constater que malgré les réalités mises en place les germes d'utopie ne sont pas absents des réalisations. Il s'agit par exemple de Biosphère II $^{1}$ : Biosphère II existe, mais n'est-elle pas de part en part traversée par la fiction? Et ce n'est pas là le seul exemple.

Que la et les techniques ou technologies soient toujours logées dans des discours fictifs ne surprendra que les esprits positivistes et hexagonaux toujours prêts à parier sur les bienfaits de l'innovation. Mais la technique comme discours fictif ne surprendra pas les spécialistes d'Amérique du Nord. Soumis plus que nous au formidable bombardement des techniques, de leurs idéologies ou utopies, ils sont les mieux à même de critiquer la technologie. Deux ouvrages récents qui voient la technique comme un récit, mais sans jamais théoriser ce point de vue, il est vrai, en témoignent. Dans Does Technology drive history, the dilemma of technological determinism $^{2}$, le terme de récit apparaît constamment. Les interprétations, nous dit-on, dépendent du type de récit (articles de Bimber, de Misa, de Léo Marx et de Scranton). De même la technologie comme base du progrès relève-t-elle d'un fétichisme culturel et politique, selon Smith. Ce sont les «master narrative», «organisationnal frame» et «meta narrative» qui structurent le raisonnement. Conclusion: dans le débat rationalité / complexité tout dépend du type de récit. Un autre ouvrage vient confirmer ce point de vue. Dans The intellectual appropriation of technology (1900-1939) ${ }^{3}$, le terme de «récit» abonde. Nous sommes aux anti-

* Pour des développements plus amples et systématiques sur ce thème, voir notre ouvrage Technique et idéologie, un enjeu de pouvoir, Paris, Le Seuil, 2002.

1 Voir Lucien Sfez, La santé parfaite, Le Seuil, 1995. C'est dans ce livre que nous posons l'idée d'utopie réalisée. Voir aussi Georges Balandier, Le grand système, Fayard, 2001. Biosphère II était la reconstitution, sous hangar de verre entièrement clos, des cinq principaux biomes de l'humanité, avec huit humains qui y vécurent deux ans.

2 Sous la direction de Merritt Rol Smith et Leo Marx, M.I.T. Press, 2ème éd., 1998.

3 Sous la direction de Mikael Hard et Andrew Jamison, M.I.T. Press, 1998. 
podes de l'idée de la technique ou technologie comme chose. Et d'ailleurs ce n'est jamais la chose en elle-même qui séduit, mais les discours tenus à son sujet.

Que la fiction de la technique nous séduise, cela ne fait aucun doute: nous n'avons qu'à prendre la mesure de nos nouveaux fétiches, de l'engouement qu'ils suscitent, et des discours pléthoriques qui les accompagnent comme autant de contes merveilleux. Entretenue par ces discours, la fiction de la technique se déploie, envahit les champs les plus divers, prend toute la place (regardez les pages consacrées aux nouveaux médias dans la presse).

Mais ces discours ont un autre rôle, plus important que celui qui consiste à promouvoir ou à vilipender, celui de substituer à la fiction de la technique le sérieux de sa prétendue objectivité. En se réclamant de l'histoire, de l'épistémologie, de la socio-anthropologie, de l'éthique ou de la philosophie, ce qui est mis en place est un discours direct, des choses elles-mêmes... comme s'il ne s'agissait pas d'intermédiaires, de distance. Comme si quelque chose comme le progrès existait réellement, en dehors des lignées dont on parle. Tous ces discours sont alors chosistes, ne font aucune différence entre choses et signes, et les prennent et se prennent pour argent comptant. D'objet du discours, la technique devient discours de l'objet. Bruno Latour ne nous propose-t-il pas un «parlement des choses » ${ }^{4}$ ?

Ce chosisme est lié à un déterminisme strict. L'objet technique fascinant devient le centre du monde et des sociétés. A chaque technique correspondrait un type de civilisation. McLuhan avec sa distinction des trois âges (tribal, scribal, néo tribal / électronique) réénonce en forme d'Auguste Comte les trois phases de la technique énoncées par Lewis Mumford, qui reprenait ici Patrick Geddes, luimême inspiré par Kropotkine. Comme l'observe James Carey: «Toutes les valeurs qui ont été prêtées à l'électricité et à la communication électrique - jusqu'à l'ordinateur, le câble et la télévision par satellite - le furent d'abord au télégraphe avec un identique mélange de fantaisie, de propagande et de vérité $»^{5}$. On pourra poursuivre cette observation humoristique jusqu'à la période contemporaine car McLuhan, suiveur de quelques-uns, a été pillé à son tour par des épigones. Un trait caractérise toute cette littérature: sa généralité. La technique comme fil à couper le beurre. Comme organisatrice des sociétés. Discours d'autant plus confortablement asséné qu'il n'est pas réfutable ${ }^{6}$.

Dernière absurdité en date: les camps de concentration nazis, c'est la faute à IBM et à ses cartes perforées, aux enregistrements et aux trieuses fournies par IBM aux nazis. La dernière décennie du millénaire a été à ce point soumise au matraquage, à la propagande sommaire du déterminisme technicien (Internet change le monde, ou, avec les biotechnologies, l'avenir est aux haras humains) qu'on peut faire grand tapage avec cette absurdité. Alors le rail aussi était la cause des trains de la mort? ${ }^{7}$

Dans Nous n'avons jamais été modernes, La Découverte, 1991, p. 194 et sq.

Voir James Carey, «McLuhan, généalogie et descendance d'un paradigme» in Quaderni n 37 , hiver 1998-99, McLuhan, trente ans après (numéro dirigé par Pascal Durand), p. 111.

6 Ce simplisme déterministe n'est jamais pratiqué par des auteurs sérieux tels que Elisabeth Eisenstein, Jack Goody ou Philippe Breton.

7 Un démographe comme Hervé Le Bras et une historienne comme Annette Wiewiorka ont fait justice de ce simplisme. Voir Hervé le Bras, «Une illusion technique» et Annette Wierwiorka, «Un 
Autre caractéristique de ces discours: au chosisme déterministe s'ajoute le fétichisme. Ce fétichisme de la technique se manifeste ainsi: il découpe la technique dans le champ de la production. Séparée initialement du procès de production pour le bénéfice de l'analyse, la technique reste séparée à la fin. On nous fait prendre alors la partie pour le tout, la technique pour la production, la production pour l'économie toute entière. C'est ainsi que les dites «nouvelles technologies» sont prises pour toute la technique, pour toute l'économie et la «nouvelle économie» (celle des nouvelles technologies) est censée tirer l'ancienne économie, voire à terme se substituer à elle. Rodomontades habituelles du technicisme, totalement démontées, dans leurs conséquences économiques, par Bernard Paulré ${ }^{8}$. Il n’y a pas plus de «nouvelle économie» qu'il n'y a de nouvelles technologies: Internet n'est qu'un bidouillage astucieux de l'ordinateur sur le téléphone, et sur la télévision bientôt. Autant de «vieilles» technologies, d'autant que le bidouillage en question date déjà des années 60 et $70^{9}$. Mais les technicistes sont des publicitaires avertis: «nouvelles technologies» sonne mieux qu'anciennes techniques et «nouvelle économie» interpelle davantage que marché des ordinateurs, du téléphone et de la télévision. Le procès de ces présentations de réclame repose très clairement sur une opération fétichiste qui fait des «nouvelles » technologies le fondement à venir de l'économie, du progrès et de la société du futur dans sa globalité. Partie pour le tout.

Obéissant à de telles consignes, chosistes, déterministes et fétichistes, ces discours, bien malgré eux, ne font que renforcer la fictivité de ce qu'ils tentent de réifier.

On peut risquer cette affirmation que des hommes politiques de tous bords et de tout premier plan assènent avec évidence des vérités trop simples sur les changements idylliques suscités par la technique, remplaçant les principes les plus éprouvés de l'organisation politique par quelques slogans publicitaires chers à Bill Gates et à Nicolas Négroponte. Sans compter les médias qui n'avaient pas attendu les hommes d'Etat pour amplifier ces thèmes jusqu'à l'obsession, et parfois au délire.

Si nous nous efforçons de saisir la logique qui sous-tend le mode fictionnel, nous nous trouvons face à des traits beaucoup plus précis et à un certain nombre de questions. La question du vraisemblable et de la crédibilité, la question d'une méthode commune à la fiction et à la technique, inventive dans le cas de la fiction, de recherche et d'innovation dans le cas de la technique, et enfin la question des rapports de la fiction avec la réalité socio-historique. Commençons par la question du vraisemblable. Et pour l'illustrer, travaillons sur l'affaire Marbot.

beau sujet gâché» dans Le Monde du 13 février 2001, à propos du livre d'Edwin Black, IBM et l' holocauste, Robert Laffont, 2001.

8 Voir Quaderni n $^{\circ}$ 40, hiver 1999-2000, Utopie 1 : la fabrique de l' utopie, son article intitulé «La new economy: enjeux et limites », pp. 11-22, et Quaderni $\mathrm{n}^{\circ} 42$, automne 2000, Utopie 2 : passages et apocalypse, son article intitulé «L'utopie néo-libérale de la new economy», pp. 55-74.

$9 \quad$ Voir Alain Le Diberder, Abécédaire du cybermonde, La Découverte, 2000. 


\section{I) LA QUESTION DE LA VRAISEMBLANCE}

\section{Marbot ou la fiction déçue ${ }^{10}$}

L'écrivain allemand Wolfgang Hildesheimer publia en 1977 une biographie de Mozart, intitulée Mozart ${ }^{11}$, ouvrage bientôt très connu et reconnu. Le même auteur publia en 1981 Marbot. Eine biographie ${ }^{12}$, biographie intellectuelle d'un spécialiste anglais d'esthétique et d'histoire de l'art, né en 1801, mort en 1830. Cet éminent spécialiste «voyageur infatigable» nous dit Schaeffer (p. 133) rencontra les figures culturelles les plus importantes de son temps: Goethe, Byron, Shelley, Leopardi, Schopenhauer, Turner, Delacroix (qui l'a dessiné au crayon, le dessin figurant dans l'ouvrage). Goethe l'admirait ainsi qu'en témoigne une lettre d'un ami de Goethe et un passage des Entretiens avec Eckermann. Marbot disparaît en 1830. Suicide? On ne sait. On apprit après sa mort, d'après des papiers personnels, qu'il avait entretenu une liaison incestueuse avec sa mère Lady Catherine Marbot. Sa vision du monde était fort pessimiste avant même de rencontrer Schopenhauer, plus encore après cette rencontre. «Lors de la sortie du livre, une partie des critiques allemands félicitèrent Hildesheimer d'avoir sorti de l'oubli une figure historique fascinante, non seulement par sa vie tragique, mais tout autant par ses théories esthétiques $»^{13}$.

Le seul problème c'est que Sir Andrew Marbot n'a jamais existé et que «malgré l'indication générique qui orne sa couverture, Marbot est une biographie imaginaire, un texte de fiction ${ }^{14}$. La seule consultation de l'Encyclopedia Britannica ou des Entretiens avec Eckermann, aurait permis de le reconnaître. Pour comprendre que cette fiction ait fonctionné comme leurre, Schaeffer propose d'analyser de près les moyens mis en œuvre par l'auteur qui ont abouti à l'invisibilité de la fiction. Il les énonce en quatre points: «le contexte auctorial, le paratexte [ou ensemble des indices autres qu'intratextuels par lesquels l'auteur ou l'éditeur oriente la lecture de son texte ${ }^{15}$, la mimesis formelle ${ }^{16}$, c'est-à-dire l'imitation énonciative de la biographie, et la contamination de l'univers historique (référentiel) par l'univers fictionnel $\gg{ }^{17}$.

Cette anecdote, analysée finement par Schaeffer, met l'accent sur les conditions de possibilité d'une fiction reconnue comme telle. Dans le cas du Marbot, ces conditions ne sont manifestement pas respectées, mais laissées de côté, gommées. Résultat: ce qui est fiction pour son auteur n'en est pas une pour ses lec-

10 Sur le même thème, on peut citer la biographie imaginaire de Ronceraille par Claude Bonnefoy, Le Seuil, 1978. Nous nous inspirons ici du récit que Jean-Marie Schaeffer fait de cette affaire dans son ouvrage Pourquoi la fiction?, Le Seuil, 1999.

11 Mozart, Suhrkamp Verlag.

12 Marbot. Eine Biographie, Surhkamp Verlag, trad. française sous le titre de «Sir Andrew Marbot», Stock, 1984.

13 Schaeffer, op. cit.

14 Schaeffer, op. cit., p. 134.

15 Ici Schaeffer renvoie à Gérard Genette et à son livre Seuils, Le Seuil, 1987.

16 Ici Schaeffer renvoie à Michal Glowinski et à son article dans Poétique n 72,1987 , «Sur le roman à la première personne », pp. 497-507.

17 Schaeffer, op. cit., pp. 136-137. 
teurs. Ce disfonctionnement est éminemment instructif, car il montre bien la forte ambiguïté du vraisemblable et son difficile maniement: tantôt penchant vers la fantaisie pure (le fantastique, le merveilleux), tantôt se rapprochant de la réalité ou ce qui est donné et reçu pour tel au point de s'y confondre. La leçon est d'importance car elle nous permet d'envisager des types de fiction qui se situent aux limites: aux limites du fantastique dans un cas, aux limites de la réalité dans l'autre. Dans ce second cas, la fiction a raté son entrée dans le vraisemblable, elle joue la proximité, voire s'identifie totalement avec ce qu'elle a pour mission de simuler; elle a ainsi valeur de vérité pour le lecteur.

On voit combien la vraisemblance est quelque chose d'extrêmement difficile à manier; on perçoit parallèlement que c'est pourtant de cette vraisemblance que dépend le fait qu'on reconnaisse qu'il y a fiction. Et, à l'inverse, c'est du défaut de vérisimilarité que dépend le fait que la fiction soit prise pour la réalité. En effet, dans le domaine de la fiction, le contraire de la vraisemblance n'est pas l'invraisemblable, mais le trop semblable. Dans le cas de Marbot on perçoit très bien que ce qui se veut fiction ressemble par trop à une biographie bien documentée, œuvre d'un érudit tout à fait sérieux, et échoue ainsi à se constituer en fiction.

Deux nécessités: s'approcher assez de la réalité qui est celle de notre vie quotidienne pour être crédible, et s'en éloigner assez pour introduire cette brèche ou cette respiration qui permet à l'imagination du lecteur ou du spectateur de pénétrer et de vivre pour quelques courts instants dans un monde parallèle. Ce parallélisme de la fiction et de la réalité constatée permet de passer d'un monde à l'autre, tantôt au bénéfice de la réalité, le lecteur retourne alors à la réalité avec un sentiment de soulagement («Heureusement, ce n'est pas comme ça dans la vraie vie»), tantôt au bénéfice de la fiction ( «Si ça pouvait vraiment être comme ça...»).

\section{II) LOGIQUES DE FICTION, LOGIQUES DE TECHNE}

Il peut sembler étrange d'introduire le vraisemblable dans la définition de la technique: celle-ci n'est elle pas conçue généralement comme à l'opposé d'une fiction? Ne possède-t-elle pas tous les traits qui en font une réalité? Voire même une réalité, lourde, souvent contraignante, une présence qui nous enveloppe de toutes parts?

Et pourtant nous formons le projet de montrer que maintes caractéristiques de la fiction vraisemblable peuvent être appliquées à la technique.

Le premier trait de ressemblance qui se présente à nous est l'ouverture de la fiction et de la technique aux divers mondes «possibles». La fiction propose en effet au lecteur un ou des mondes "possibles», dotés chacun d'une cohérence propre, d'une logique des situations (elles ont une logique de développement temporel), des personnages (ils ont une ligne de conduite) et des comportements spécifiques. Les mondes proposés par la fiction sont dotés d'une unité qui les rend «habitables» et en fait des exemplaires ou des échantillons de "possibles ». 
Les mondes possibles sont des mondes «inventés », construits parallèlement aux nôtres, et où nous pouvons projeter des doublures, munies de caractéristiques solides, qui ne se démentent pas ${ }^{18}$.

Or il est indéniable que la technique nous offre aussi des perspectives cohérentes dans des mondes possibles, perspectives qu'elle entend bien réaliser. L'innovation (ou l'invention) technique projette au devant d'elle des fictions vraisemblables. Elle leur est consubstantielle. Fiction et technique sont donc alliées.

Un second trait qui renforce cette alliance est l'obéissance de la fiction comme de la technique à une certaine croyance communément partagée à une époque donnée. Il existe en effet une communauté de croyances et d'usages, à laquelle la fiction doit souscrire si elle veut passer pour crédible, si elle veut être entendue. Il en est de même pour les innovations de la technique: elles doivent correspondre à une possibilité envisageable par les populations, elles doivent être comprises de la même manière que la fiction doit être crédible; la technique doit passer par une sorte de consensus, par une communauté des usages pour fonctionner et se réaliser avec succès. Faites de strates diverses, composées entre elles, ces communautés intègrent tout à la fois mythes identitaires, histoire générale du monde - et particulière - de la nation, plus particulière encore de la famille ou du clan; les religions et les morales sont aussi présentes ainsi que les expériences transmises de boucheà-oreille, recommandations et interdits touchant la nourriture et les attitudes. Plus étroites que les «civilisations » et plus larges que les «cultures», ces communautés d'usages et de croyances dessinent un cercle à ne pas transgresser, sous peine de rompre la liaison de la fiction avec la crédibilité, et la technique avec ses réalisations. Ce trait n'a pas échappé aux historiens des techniques, qui remarquent que tel moulin, telle ingénieuse machine ne pouvaient être «inventés » à tel moment dans tel pays, mais étaient possibles à tel autre. Ou bien qu'il y eut bien invention, mais dénuée de suite. Ou encore que malgré les circonstances favorables (aux yeux de l'historien), l'invention passa par-dessus la tête des ingénieurs. Les composants de l'aventure technicienne offrent un nombre infini de combinaisons.

\section{III) FICTION SOCIO-HISTORIQUE ET UTOPIE}

Nous présenterons ici deux types de fiction: la fiction socio-historique et la fiction sociale, appelée quelquefois «utopie».

\section{La fiction socio-historique}

L'histoire, nous dit Jean-Pierre Faye ${ }^{19}$, rend compte, c'est-à-dire «demande conte»; elle narre, plus encore: elle raconte les narrations qui rendent et ont rendu possible les événements. «Les récits doivent changer la face des nations», met-il en exergue de sa Théorie du récit, il y a un «effet de récit», une production d'actions par le récit, et à tout le moins une production d'identité.

\footnotetext{
18 En ce sens, la logique de la fiction relève de l'existence de ces mondes possibles réclamés par la logique analytique, et pour lesquels la question se pose de l'identification ou de la non-identification d'un individu $\mathrm{X}$ du monde $\mathrm{A}$ dans un des mondes possibles $\mathrm{B}$.

19 Jean-Pierre Faye, Théorie du récit, Hermann, 1972.
} 
La liaison entre les individus se constitue, en effet, sur le fil d'un récit historique. Liant passé archaïque, présent et futur, l'histoire constitue à elle seule un «grand récit» ou méta-récit, qui est l'aliment de base des individualités particulières et de l'identité collective; l'histoire arrime nos identités singulières à un modèle éprouvé, elle nous donne un terrain où ancrer nos opinions, nos jugements, voire nos certitudes; l'histoire enfin s'offre à nous dans le mode du récit, avec ses composants fictifs que nous percevons comme une réalité attestée.

Rappeler que les rois de France aient eu besoin de mémorialistes et d'historiographes, c'est-à-dire de narrateurs, pour attester de leur existence en tant que rois, c'est affirmer que le réel a besoin de fiction pour se constituer en tant que réel. Ce qui est alors raconté, mis en scène et transcrit au moment où les événements se produisent devient une sorte de vérité inscrite pour les générations futures. On oublie qu'il s'agit de récit, avec tout ce que ce genre comporte d'indéterminé et d'artifice, c'est-à-dire de fabrication, pour se saisir de la capacité du récit à forger une unité vraisemblable ${ }^{20}$. Le récit historique exige que l'on trie et compose les événements entre eux, en sorte que l'ensemble corresponde à un récit idéal: vraisemblable dans son déroulement, et garanti dans les éléments qui en composent la trame, tels que personnages et dates, ce sont là des indices de réalité.

Or, ces remarques sur le récit que fabrique l'histoire sont valables, tout aussi bien, pour ce qui nous intéresse ici, pour la technique.

Nous avions déjà remarqué comment les histoires de la technique proposent une vision «objectiviste» de l'avancée technique. Objectiviste, car ce qui nous est conté ce sont des énumérations d'objets, se succédant le long d'un fil narratif. De l'objectivisme de la narration d'objets, on passe sans coup férir à l'objectivité, car il semble toujours qu'une description soit neutre, et prenne un point de vue photographique sur une réalité qui, dès lors, ne peut plus être sujette à suspicion. L'objet produit de l'objectivité, pour ainsi dire... Mais si nous reprenons l'hypothèse de l'histoire comme récit et fabrique, comme fiction, aussitôt nous sommes pris d'un doute. $\mathrm{Ne}$ s'agit-il pas d'une sorte de constitution, de fabrication d'une entité, la technique, par le moyen d'un récit historique à valeur de preuve, qui se sert des outils propres à l'historien pour accréditer une version des événements ayant eu lieu?

Arrêtons-nous un instant sur ces points: si l'histoire fournit aux individus un terrain d'identification, le récit de leur propre histoire en tant que nation, peuple ou communauté, elle établit aussi, parallèlement, nations, peuples ou communautés comme entités. Elle les dote d'une vie propre, leur fournit naissance et développement, disparition et mort, sur le modèle du vivant. Nous croyons tous à l'histoire de notre pays, nous en apprenons les éléments à l'école, les trouvons inscrits dans la pierre, les cités, les grottes, les inscriptions, les monuments, les fondations humaines, et ne les mettons généralement pas en doute, bien que nous n'ayons pas ou peu d'accès à leurs sources. Ces événements ont eu lieu ${ }^{21}$, nous dit-on, mais c'est par des médiations successives que nous en avons des preuves.

20 Gabriel Bonnot de Mably, Observation sur l'histoire de France, De l'Imprimerie privilégiée, 1791.

21 Jean-Marc Levy-Leblond nous dit de même que dans le domaine scientifique ce qui est fiction à une époque devient réalité figée pour l'époque suivante. Voir son essai Aux contraires, Gallimard, 1996, p. 396. 
La technique, si nous suivons cette voie d'analyse, est créditée, elle aussi, en tant qu'entité, domaine ayant sa vie propre, par un récit qui narre la succession d'objets produits, qui ont eu lieu et que la fiction historique met en scène. Mais contrairement à l'histoire d'une nation, ou d'un peuple, qui sont des notions abstraites, la technique se manifeste sous forme de ces objets qui sont indispensables à notre vie actuelle. Ici la fiction historique qui a pour objet la technique est plus qu'identificatrice, elle indexe et désigne la réalité dans sa présence immédiate et se sert de cet appui pour dévoiler l'avenir.

En effet, l'histoire, telle qu'elle nous est contée à l'école et plus tard, pour les amateurs, est un récit dont nous pouvons nous détacher, quel que soit l'intérêt qu'il suscite («Cela a été ainsi ou ainsi», disons-nous, revenant ensuite à nos affaires).

L'histoire des techniques, en revanche, si elle reste souvent récit et légende (la longue saga des moulins, celle des instruments de tirage ou de roulage, en témoigne) est cependant aussi, sur un autre versant, fortement attirée vers la description d'une réalité présente, enveloppante, qui non seulement atteste que nous sommes bien dans cette histoire-là, mais encore que cette histoire nous mène toujours plus loin dans son propre développement, indépendamment de nos souhaits. En somme, une certaine histoire des techniques sert d'indexation: «Voilà où nous sommes, voilà ce qui nous attend», dit-elle.

Ce qui est fiction historique, histoire structurale avec ses hiérarchies et ses feed back, quand elle est racontée par Bertrand Gille, devient, sous d'autres plumes de certains, attestation ou affirmation déterministe et linéaire. Le vraisemblable fictif y tourne au réel attesté, ayant pour critère de fiabilité la réalité de l'objet présent et ses usages actuels toujours en extension. Nous nous trouvons alors dans le cas de cette fiction qui ne joue pas le jeu et dénonce les principes même de la fictivité, comme faisait tout à l'heure la fiction du Marbot, dont l'auteur, bien qu'il ne l'ait ni pensé, ni prévu, avait détruit la structure fictive. De même l'histoire des techniques ou des objets techniques, qui est récit, se transforme quelquefois et de plus en plus souvent, en source de certitude pour une vision totalitaire de la technique.

Et sans doute est-ce là que l'on voit fonctionner le mécanisme de transformation qui fait d'une histoire des techniques une mémoire nationale, se substituant à l'histoire politique. La technique, ainsi convoquée par l'histoire au rang d'une politique, les gouvernants peuvent alors tabler sur sa capacité à rassembler les citoyens, à représenter la totalité de la vie de la nation, et peuvent, croient-ils, compter sur son développement exponentiel pour développer eux-mêmes leur propre pouvoir.

\section{Utopie et technique}

Il existe d'autres fictions qui ont leur part dans la construction d'un grand récit de la technique: ce sont les fictions dites «utopiques». Vecteurs d'identité, d'homogénéisation et d'acculturation, elles visent l'intégration d'éléments hétérogènes dans un ensemble narratif qui les distribue en unités conjointes.

Pour en donner un exemple qui ne nous éloignera pas fondamentalement de nos préoccupations actuelles, prenons Saint-Simon et son Nouveau christianisme ${ }^{22}$. Il

22 Claude-Henri de Saint-Simon, Le nouveau christanisme, Euvres, tome III, Anthropos, 1966. 
s'agit là de «fictionner» une société complète, économie, socio-politique liées: répartition des biens, distribution des tâches, technique appropriée, morale civique et religion laïque. Organisation du travail en réseaux territoriaux et application d'une loi chrétienne de l'égalité entre frères permettraient de parvenir à cette communication générale qui devrait profiter aux plus pauvres.

On voit combien c'est là un méta-discours ou grand récit, qui lie l'état présent d'une société inégalitaire et un projet de recomposition et de redistribution de l'ensemble. Les images instauratrices ont été de son propre aveu, le système de la pompe avec ses va et vient (ou distribution alternée), la machine à vapeur avec son régulateur à boules, et l'organisme vivant qui intègre ces différents mouvements: le cœur comme pompe et les réseaux sanguins comme distribution autorégulée. Images instauratrices que la fiction saint-simonienne assemble en un tout pour une longue période. Comme le souligne Pierre Musso, cette fiction datant du XIXème siècle joue encore et plus que jamais un rôle aujourd'hui ${ }^{23}$.

\section{Science-fiction utopiste}

Au-delà de Saint-Simon, il nous faut signaler l'évolution contemporaine des utopies technologiques vers la science-fiction et indiquer qu'un genre est né24: la science-fiction utopiste (ou SFU), qu'on peut résumer par deux traits.

(1) Une compression du temps. Au lieu de projeter en effet une image d'avenir à partir du présent, la SFU construit l'avenir à partir d'un passé hypothétique (le dinosaure) immédiatement raccordé au futur. Car si le présent peut prêter à contestation, ni un passé aussi lointain, ni un avenir tout aussi lointain ne peuvent être jugés sur pièces: ils restent donc tous les deux illusoires, mais s'entretiennent mutuellement de leur vraisemblance. Ce qui compte pour la SFU c'est cette hésitation entretenue entre rêve et réalité: si les dinosaures ont existé, ce qui est incontestable, on ne connaît pas cependant leurs conditions concrètes de vie et de mort, alors la réalité virtuelle vient à se substituer à notre ignorance. Les deux extrêmes du temps, passé et futur, se rejoignent dans un même sentiment de demi-croyance. Le présent a disparu, «compacté» en quelque sorte dans un temps dont la durée vécue est absente.

(2) Une hybridation d' images. Les figures du discours qu'employaient les utopies classiques, ont acquis avec les outils technologiques une sorte de réalité: elles sont visibles, elles sont des images d'abord, auxquelles le texte est surajouté (alors que dans l'utopie classique l'image venait à l'esprit du lecteur d'après le texte) elles ne peuvent donc, pense-t-on, tromper.

23 Sur cette fiction sociale voir Pierre Musso, Télécommunications et philosophie des réseaux, PUF, 1997.

24 Voir par exemple: Etonnants voyageurs, utopies S.F., anthologie présentée par Michel Le Bris avec la collaboration de Jacques Chambon, Hoëbekeim, 2000. Dans ces textes, c'est visiblement la technique qui fait le passage de l'utopie vers la science-fiction. Dans le même sens, voir «Utopie et science-fiction » par Serge Lehman, Le magazine Littéraire, mai 2000, p. 31. 
C'est un lieu commun que de constater l'emprise et l'empire de l'image sur notre monde contemporain. Mais il faut bien comprendre les effets de cet entassement d'images: il installe un troisième monde entre le réel et l'imaginaire, un «demi-monde ${ }^{25}$ si l'on veut, et où il est impossible ou du moins difficile de faire le départ entre nature et artifice (les tamagotchis en sont un exemple). Nous vivons alors dans la fiction, dans un univers mi-apparence mi-réalité; films, téléfilms et documentaires aidant, nous ne démêlons qu'à grand peine ce qui appartient à la science ou à la fiction, au réel ou à l'illusion. Le monde du Net, devenant le monde tout court et non plus un outil pour opérer sur le monde, projette son illusion sur l'ensemble de nos croyances. En fait, même si par ailleurs un certain bon sens nous dictait la prudence, le vraisemblable est à la mesure de ce que nous voulons croire.

\section{Fiction technique et séduction : l'amour de la technique}

Que la fiction soit ainsi l'alliée de la technique au point que la technique ne puisse s'exporter vers le champ social sans son aide, et qu'intimement, elle participe de la logique fictionnelle, non seulement nous en avons des preuves multiples, mais encore nous pouvons percevoir à quel point l'adhésion au monde de la technique, que j'appellerai «désir de la technique», est liée à la séduction opérée par la fiction.

Sur ce point, nous pourrions énumérer ici un nombre incalculable d'ouvrages de fiction qui orientent le lecteur vers un monde où la technique est omniprésente. Mais il nous suffira d'évoquer, par exemple, la production d'un Jules Verne pour saisir la portée d'une telle omniprésence et pour mesurer la séduction que ses récits ont exercée.

Autre exemple de notre temps, les récits d'apocalypse: si tous les ordinateurs du monde s'arrêtaient! Philippe Quéau s'est fait une spécialité de cette prophétie, tandis qu'on a cru dur comme fer au bug informatique mondial au passage de l'an 2000. Panne fictive qui donne la mesure de notre amour de la technique. Une fois encore le «désir de la technique» passe par la fiction.

Communication, technologies et pouvoir

Université de Paris I Panthéon-Sorbonne

25 Et ceux qui le pratiquent sont des «demi-mondains». 